\title{
Imaging and atrial fibrillation: A new paradigm for precision targeting of AF?
}

\author{
Mina K. Chung, MD, ${ }^{\mathrm{a}, \mathrm{b}}$ Sojin $Y$. Wass, $M \mathrm{D}^{\mathrm{a}, \mathrm{b}}$ and Manuel Cerqueira, $\mathrm{MD}^{\mathrm{a}, \mathrm{c}, \mathrm{d}}$ \\ a The Department of Cardiovascular Medicine, Heart \& Vascular Institute, Cleveland Clinic, \\ Cleveland, $\mathrm{OH}$ \\ b Department of Cardiovascular \& Metabolic Sciences, Lerner Research Institute, Cleveland \\ Clinic, Cleveland, $\mathrm{OH}$ \\ c Department of Nuclear Medicine, Imaging Institute, Cleveland Clinic, Cleveland, $\mathrm{OH}$ \\ d Department of Cardiovascular Imaging, Heart and Vascular Institute, Cleveland Clinic, \\ Cleveland, $\mathrm{OH}$
}

Received Sep 20, 2018; accepted Sep 21, 2018

doi: $10.1007 / \mathrm{s} 12350-018-01496-9$

\section{See related article, pp, 1501-1512}

Atrial fibrillation (AF) is the most common sustained arrhythmia seen in clinical practice. Recent data from the Framingham Heart Study have shown a lifetime risk of $\mathrm{AF}$ now reaching $37 \%$ in the average individual over age 55 and even higher in individuals with elevated risk factors. ${ }^{1}$ Two of the recognized risk factors include older age and obesity. With our aging population and increases in obesity, the prevalence of $\mathrm{AF}$ is projected to continue to increase dramatically in the future. The etiology for AF is multifactorial and includes genetic, structural and local cardiac electrical and biochemical physiology, as well as potential inflammatory and metabolic factors that can influence the electrical activity of the heart. To date, cardiovascular imaging for characterization and treatment of $\mathrm{AF}$ has been performed primarily with anatomic techniques such as echocardiography, cardiac CT, and cardiac magnetic resonance imaging. Radionuclide techniques, which are capable of assessing local cardiac physiology, have not been used extensively, but may have a role in the classification and management of AF based on our evolving understanding of the mechanisms of AF. The search for effective prevention and treatment of AF will

Reprint requests: Mina K. Chung, MD, The Department of Cardiovascular Medicine, Heart \& Vascular Institute, Cleveland Clinic, 9500 Euclid Avenue, J2-2, Cleveland, OH 44195; chungm@ccf.org J Nucl Cardiol 2020;27:1513-6.

$1071-3581 / \$ 34.00$

Copyright (c) 2019 American Society of Nuclear Cardiology. be more successful if focused on modifiable risk factors with therapies targeting precisely characterized subsets of $\mathrm{AF}$.

The search for effective upstream therapies for the treatment or prevention of AF has seen dramatic changes over the past two decades. In the 1950s, Moe and Abildskov postulated an atrial substrate based theory whereby multiple random reentrant wavelets, which were more frequent in large atria, resulted in $\mathrm{AF}^{2}$ The ground-breaking report by Haissaguerre and colleagues ${ }^{3}$ in 1998 demonstrated that triggers originating in the pulmonary veins could initiate AF. This revolutionized our concept of AF pathophysiology and led to our current explosion of ablative therapies targeting pulmonary vein isolation. In parallel to the development of these ablative approaches targeting the initiation of AF were studies focused on the atrial electrical and structural remodeling that can occur with and potentially promote the progression of $\mathrm{AF}$ to more persistent forms. Yet the prevention of $\mathrm{AF}$ progression and cure of persistent $\mathrm{AF}$ have remained a bane of ablation and medical therapies.

Early studies suggested inflammatory and oxidative stress as key contributors to structural remodeling of the atria in AF. Rapid atrial and ventricular stimulation can lead to calcium overload, inflammatory, metabolic, and oxidative stressors that could promote electrical and structural remodeling. The inflammatory hypothesis was fueled by the high incidence of AF after cardiac surgery, reaching $25 \%$ to $50 \%$ after coronary or valvular operations. After surgery, early increases in IL- $1 \beta$ and then IL-6 are followed by elevations in C-reactive protein (CRP) and complement-CRP complexes, peaking on postoperative days 2 to 3 , coincident with the peaking of postoperative $\mathrm{AF}$ incidence. ${ }^{4}$ In non-postoperative $\mathrm{AF}$, 
inflammatory infiltrates, myocyte necrosis, and fibrosis were also reported in atrial biopsies. ${ }^{5}$ Our group then studied ultrasensitive CRP, a marker of systemic inflammation, and found levels to be elevated in AF with stepwise CRP elevation associated with higher AF burden. ${ }^{6} \mathrm{CRP}$ was also associated with the presence of AF and predicted patients at increased risk for future development of AF in the Cardiovascular Health Study. ${ }^{7}$

Such studies provided rationale for the study of putative upstream therapies for $\mathrm{AF}$ that targeted inflammatory, fibrotic, calcium overload, and oxidative stress pathways using statins, steroids, ACE inhibitors, ARBs, and omega-3 fatty acids. However, while these drugs appeared to be associated with lower AF in the conditions for which they were originally developed, like coronary artery disease, heart failure, or hypertension, when studied specifically for $\mathrm{AF}$ in randomized trials, results were disappointing. Notable exceptions were steroids and statins for reduction of postoperative $\mathrm{AF}$, a clear inflammatory subset of AF. Nevertheless, the disappointing nature of the studies in non-postoperative AF led to greater uncertainty as to whether inflammation or AF was the chicken or the egg.

More recently, the study of genetics has led to perhaps the ultimate upstream approach to studying AF. Multiple large consortia have collaborated to perform genome-wide association studies (GWAS) over the past 10 years, culminating this year in over 100 loci associated with AF risk. ${ }^{8}$ The top AF locus is on chromosome $4 \mathrm{q} 25$, near PITX2, a gene implicated in formation of the pulmonary veins. Several top genetic loci are active early in cardiac development. However, AF generally does not develop until 5 to 7 decades later. ${ }^{9}$ Studies of human left atrial appendage tissue removed at the time of cardiac surgery have allowed us to study the adult atrial transcriptome in order to attempt to identify functional links between genetic variants ${ }^{10}$ and $\mathrm{AF}$ subsets $^{11}$ with gene expression patterns. Such studies have identified an overwhelmed or reduced transcriptional response to cell stress, including inflammatory, oxidative, metabolic, mitochondrial, and protein handling stressors. ${ }^{11}$ We have proposed a dual risk model for the perfect storm for AF, where in patients with genetic susceptibility to pulmonary vein triggers from genes active in development, inadequate transcriptional responses to cell stress later in life predispose to AF. Identification of $\mathrm{AF}$ phenotypes, perhaps characterized by type of stress, may lead to targetable therapies in subsets of AF. A major thrust of our group's research is focusing on identifying subsets of AF through a multiomics approach.

The current study in this journal potentially introduces PET/CT F-18 FDG imaging of atria and surrounding epicardial fat that may be particularly relevant to the process of identifying patients with particular AF phenotype subsets that might be studied for targeted therapies. ${ }^{12}$ This was a retrospective study in patients with whole body PET/CT scans presumably for known or suspected cancer that over a 7-year period identified 48 patients with AF. This cohort included a relatively small number of patients, and the $\mathrm{AF}$ itself was not well characterized and ranged in duration from 1 to 66 months. Nevertheless, these patients were compared to 22 patients that were "normal," i.e., no $\mathrm{AF}$. In the AF group, 32 had persistent $\mathrm{AF}$ and 16 paroxysmal. Interestingly, $17 \mathrm{AF}$ patients were found to have increased atrial FDG uptake, all with persistent AF.

Given the small numbers and the suboptimal characterization of AF these results are not definitive, but are suggestive that this technique may have a role in identifying subsets of AF patients where inflammation may play a role. Other limitations include the need to use CT attenuation maps to localize the atrial structures due to the low counts and the relatively poor spatial resolution of PET and variability introduced by the relatively high F-18 FDG counts in the adjacent left ventricular myocardium, which is variably suppressed and capable of influencing the measured $\mathrm{SUV}_{\max }$ values.

While a recent study has implicated inflammation via inflammasome signaling as being potentially causative for $\mathrm{AF}^{13}$ caution should also be exercised in implicating imaged inflammation by FDG uptake as causative for persistent AF. The persistence of the arrhythmia itself can set up a vicious circle of atrial remodeling by mechanisms and pathways discussed above. Moreover, FDG uptake is not specific for an inflammatory process, but indicates uptake by highly metabolically active cells, ${ }^{14}$ as might be the case during the high stimulation rates seen in the atria during AF. In the subset of patients who had CRP levels, there was no correlation with FDG uptake by $\mathrm{SUV}_{\max }$, although it is not clear whether CRP or ultrasensitive CRP assays were used. These issues could be addressed by enrolling a larger number of AF patients with better characterization of the duration of and comorbid conditions associated with AF.

Epicardial adipose has been in itself associated with $\mathrm{AF}$ in several studies, ${ }^{15}$ as well as $\mathrm{AF}$ recurrence after ablation. ${ }^{16}$ Adipose tissue is known to be highly metabolically active and to secrete proinflammatory adipokines. ${ }^{17}$ Increased FDG uptake in epicardial adipose tissue was observed in this study and may turn out to be as important to stratifying $\mathrm{AF}$ patients with high inflammatory or metabolic burden as uptake in atrial tissue.

The distribution of increased uptake that seemed more prevalent in the right atrium in $82 \%$ of the $\mathrm{AF}$ patients has been previously described in patients 
scanned with FDG for cancer. ${ }^{18}$ It is uncertain whether this might reflect an artifact of the target-to-background ratio (TBR) or $\mathrm{SUV}_{\max }$ calculations with different thresholds for left vs right atria. Or as suggested by the authors this could reflect higher fibrosis burden in the left atria. In contrast, when atrial appendages showed FDG uptake in the current study, right and left atria were equally affected and may reflect the high metabolic activity of these sub-organs. Atrial appendages are also known to produce high amounts of atrial natriuretic peptide (ANP), a protein which has co-localized with atrial amyloid protein deposits and which might provoke an inflammatory response. ${ }^{19}$ Another related explanation could be that the differential uptake reflects differential metabolic effects in the atria. As noted above, our genomics studies in left atrial appendage implicated a reduction in transcriptomic responses to metabolic stress. The left atria, where AF drivers or triggers may be more prevalent, could be more susceptible than the right atria to metabolic stress in AF. A potential implication is that left to right atrial mis-match could indicate a subset of AF patients in whom metabolic interventions could be tested. Clarification of these issues requires enrollment of a larger number of patients to have adequate statistical power.

The potential future implications of being able to image inflammatory or metabolic stress in the atria or surrounding epicardial fat have potential implications for managing AF. Imaging modalities have been limited by the thinness of the atria walls, but an ability to image by PET/CT could represent a new paradigm in stratifying AF patients toward targeted therapies (Figure 1). We are not far from identifying new metabolic or antiinflammatory therapies. For example, the involvement of inflammasome activation leading to IL- $1 \beta$ activation in AF opens the door to potentially using canakinumab, the drug tested in the CANTOS trial that blocks interaction of activated IL-1 $\beta$ with IL-1 receptors, reducing CRP levels, and cardiovascular events. ${ }^{20,21} \mathrm{We}$ await subanalyses of AF effect, but an imaging modality that could detect inflammation could provide a more specific subset of patients with higher yield from not only canakinumab but other novel anti-inflammatory therapies. Interventions targeting the metabolic aspects of AF are being tested in a clinical trial Upstream Targeting for the Prevention of AF: Targeting Risk Interventions and Metformin for Atrial Fibrillation (TRIM-AF) [clinicaltrials.gov NCT03603912].

Future clarification of imaging criteria for calling elevated FDG uptake and prospective studies will be critical to adoption of PET FDG/CT for atrial imaging and use in targeting therapies. The use of novel radiotracers that can distinguish metabolic vs. inflammatory tissue has been a long-awaited goal of PET imaging and

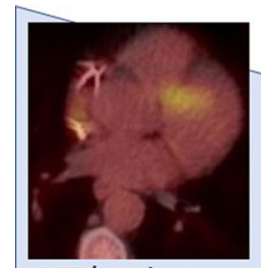

PET/Nuclear

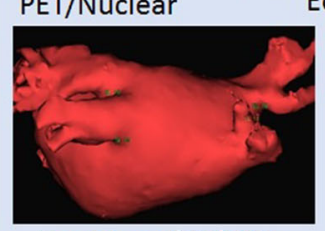

Radiology/CT/MRI

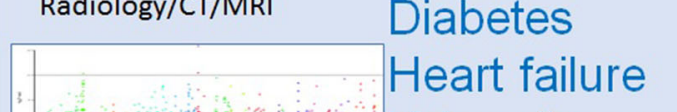

Heart failure

Obesity

Genetics

Aging

Hypertension

Diabetes

Valve disease

Genomics

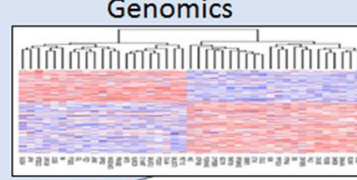

Transcriptomics/Proteomics/Metabolomics/Biomarkers
Not all $A F$ is created equal

Towards use of multimodality imaging and multi-'omics to subset $A F$ for targeted therapies

Pulmonary vein triggers

Oxidative stress

Metabolic stress

Inflammation

Atrial Fibrillation

Calcium overload

Fibrosis

Hemodynamic stress

Abnormal proteostasis

Mitochondrial dysfunction

Figure 1. Potential role of imaging, patient comorbidities and multi-omics in identification of atrial fibrillation subsets for prevention and treatment. 
will be highly applicable to imaging in patients with AF. However, we commend the authors for taking this first important step in opening a new window for potentially meaningful imaging of the atria.

\section{Disclosures}

The authors declare that they have no conflict of interest to disclose.

\section{References}

1. Staerk L, Wang B, Preis SR, Larson MG, Lubitz SA, Ellinor PT, et al. Lifetime risk of atrial fibrillation according to optimal, borderline, or elevated levels of risk factors: Cohort study based on longitudinal data from the Framingham heart study. BMJ. 2018;361:k1453.

2. Moe GK, Abildskov JA. Atrial fibrillation as a self-sustaining arrhythmia independent of focal discharge. Am Heart J. 1959;58:59-70.

3. Haissaguerre M, Jais P, Shah DC, Takahashi A, Hocini M, Quiniou G, et al. Spontaneous initiation of atrial fibrillation by ectopic beats originating in the pulmonary veins. $\mathrm{N}$ Engl $\mathrm{J}$ Med. 1998;339:659-66.

4. Bruins $P$, te Velthuis $H$, Yazdanbakhsh AP, Jansen PG, van Hardevelt FW, de Beaumont EM, et al. Activation of the complement system during and after cardiopulmonary bypass surgery: Postsurgery activation involves c-reactive protein and is associated with postoperative arrhythmia. Circulation. 1997;96:3542-8.

5. Frustaci A, Chimenti C, Bellocci F, Morgante E, Russo MA, Maseri A. Histological substrate of atrial biopsies in patients with lone atrial fibrillation. Circulation. 1997;96:1180-4.

6. Chung MK, Martin DO, Sprecher D, Wazni O, Kanderian A, Carnes CA, et al. C-reactive protein elevation in patients with atrial arrhythmias: Inflammatory mechanisms and persistence of atrial fibrillation. Circulation. 2001;104:2886-91.

7. Aviles RJ, Martin DO, Apperson-Hansen C, Houghtaling PL, Rautaharju P, Kronmal RA, et al. Inflammation as a risk factor for atrial fibrillation. Circulation. 2003;108:3006-10.

8. Roselli C, Chaffin MD, Weng LC, Aeschbacher S, Ahlberg G, Albert CM, et al. Multi-ethnic genome-wide association study for atrial fibrillation. Nat Genet. 2018;50:1225-33.

9. Go AS, Hylek EM, Phillips KA, Chang Y, Henault LE, Selby JV, et al. Prevalence of diagnosed atrial fibrillation in adults: National implications for rhythm management and stroke prevention: The anticoagulation and risk factors in atrial fibrillation (atria) study. JAMA. 2001;285:2370-5.

10. Hsu J, Hanna P, Van Wagoner DR, Barnard J, Serre D, Chung $\mathrm{MK}$, et al. Whole genome expression differences in human left and right atria ascertained by rna sequencing. Circ Cardiovasc Genet. 2012;5:327-35.

11. Deshmukh A, Barnard J, Sun H, Newton D, Castel L, Pettersson $\mathrm{G}$, et al. Left atrial transcriptional changes associated with atrial fibrillation susceptibility and persistence. Circ Arrhythm Electrophysiol. 2015;8:32-41.

12. Xie B, Chen BX, Wu JY, Liu X, Yang MF. Factors relevant to atrial ${ }^{18} \mathrm{~F}$-fluorodeoxyglucose uptake in atrial fibrillation. J Nucl Cardiol. 2018. https://doi.org/10.1007/s12350-018-1387-4.

13. Yao C, Veleva T, Scott L Jr, Cao S, Li L, Chen G, et al. Enhanced cardiomyocyte NLRP3 inflammasome signaling promotes atrial fibrillation. Circulation. 2018. https://doi.org/10.1161/CIRCULA TIONAHA.118.035202.

14. Davidson CQ, Phenix CP, Tai TC, Khaper N, Lees SJ. Searching for novel pet radiotracers: Imaging cardiac perfusion, metabolism and inflammation. Am J Nucl Med Mol Imaging. 2018;8:200-27.

15. Batal O, Schoenhagen P, Shao M, Ayyad AE, Van Wagoner DR, Halliburton SS. Left atrial epicardial adiposity and atrial fibrillation. Circ Arrhythm Electrophysiol. 2010;3:230-6.

16. Wong CX, Abed HS, Molaee P, Nelson AJ, Brooks AG, Sharma G. Pericardial fat is associated with atrial fibrillation severity and ablation outcome. J Am Coll Cardiol. 2011;57:1745-51.

17. Mazurek T, Kiliszek M, Kobylecka M, Skubisz-Gluchowska J, Kochman J, Filipiak K, et al. Relation of proinflammatory activity of epicardial adipose tissue to the occurrence of atrial fibrillation. Am J Cardiol. 2014;113:1505-8.

18. Fujii H, Ide M, Yasuda S, Takahashi W, Shohtsu A, Kubo A. Increased FDG uptake in the wall of the right atrium in people who participated in a cancer screening program with whole-body PET. Ann Nucl Med. 1999;13:55-9.

19. Rocken C, Peters B, Juenemann G, Saeger W, Klein HU, Huth C, et al. Atrial amyloidosis: An arrhythmogenic substrate for persistent atrial fibrillation. Circulation. 2002;106:2091-7.

20. Ridker PM, Everett BM, Thuren T, MacFadyen JG, Chang WH, Ballantyne C, et al. Antiinflammatory therapy with canakinumab for atherosclerotic disease. N Engl J Med. 2017;377:1119-31.

21. Ridker PM, MacFadyen JG, Everett BM, Libby P, Thuren T, Glynn RJ. Relationship of c-reactive protein reduction to cardiovascular event reduction following treatment with canakinumab: A secondary analysis from the cantos randomised controlled trial. Lancet. 2018;391:319-28. 